\title{
Bowel Perforation due to COVID-19: A Mini Review
}

\author{
Manisha V Ramani ${ }^{1}$, Rajiv K Saxena ${ }^{2}$, Chaitanya Indrani ${ }^{3}$
}

\begin{abstract}
Background: The severe acute respiratory syndrome coronavirus-2 was initially believed to be an infection of only the respiratory system. It is now known to have many extrapulmonary affections including gastrointestinal symptoms associated with high mortality rates. An unusual presentation of bowel perforation in a patient who had recently undergone cesarean delivery prompted us to report this mini review of bowel perforations attributed to coronavirus.

Case: Our patient, a 19-year-old primigravida, underwent an emergency cesarean section under spinal anesthesia. Intraoperative events and postoperative period were uneventful, and the patient was discharged on postoperative day 5 . On the 10th postoperative day, the patient presented in septic shock to the emergency department with complaints of acute epigastric pain, vomiting, loose stools, and bilious discharge from the wound site. Oral contrast computed tomography was done, which revealed a proximal jejunal perforation. The patient was taken up for laparotomy followed by bowel repair with placement of intraperitoneal drains. Immediate postoperatively, the patient developed highgrade fever for which reverse transcription polymerase chain reaction (RT-PCR)_ for COVID-19 was sent. The result for the same turned out to be positive. Twenty-four hours after the laparotomy, the intraperitoneal drains again started draining copious bilious discharge along with the deterioration of general condition of the patient. She expired on the third postoperative day.

This was followed by a thorough research of the literature into possible etiologies for bowel perforation. In this mini review, we shall be discussing various case reports to determine the cause of such unusual presentation in our patient.

Keywords: Bowel injury; Complications; COVID-19; Gastrointestinal manifestations; Jejunal perforation; Maternal death

Journal of South Asian Federation of Obstetrics and Gynaecology (2020): 10.5005/jp-journals-10006-1845
\end{abstract}

\section{BACKGROUND}

The severe acute respiratory syndrome coronavirus-2 (SARS-CoV-2) is caused by COVID-19, a new microorganism described in the family of coronaviruses. This disease, first described in late 2019, was declared a pandemic by World Health Organization on March 11, 2020. Initially, the illness was believed to be an infection of the respiratory system with the most common symptoms being fever, cough, sore throat, fatigue, and myalgia with severe cases progressing to shortness of breath, acute respiratory distress syndrome (ARDS), and life-threatening respiratory failure. ${ }^{1}$ As new studies come to light, many extrapulmonary affections of this virus have now been reported, which include gastrointestinal symptoms, myocardial infarction, renal dysfunction, liver dysfunction, and hypercoagulability. ${ }^{2}$

Possible pathophysiology for gastrointestinal injury could be explained due to selective tropism of the virus for angiotensinconverting enzyme-2 (ACE-2) receptors. COVID-19 virus enters the host cells through ACE-2 receptors. ${ }^{3}$ Apart from lung alveolar epithelium, these receptors are also expressed in enterocytes of small intestine and vascular endothelium. ${ }^{4}$ The most common gastrointestinal symptoms are abdominal pain, anorexia, nausea, and diarrhea, largely due to direct endothelial cell invasion of gut vessels by SARS-CoV-2. In severe cases, extensive tissue distribution of coronavirus releases a high number of proinflammatory cytokines like interleukin 6 (IL-6) and other acute phase reactants that activate a "cytokine storm" and cause further endothelial cell injury. The resulting endotheliitis, generalized small-vessel vasculitis, and extensive microthrombi can result in infarction, necrosis, and perforation of the bowel. ${ }^{5,6}$ Such severe cases usually have a poor prognosis with high mortality rates. ${ }^{5}$
${ }^{1-3}$ Department of Obstetrics and Gynaecology, MVJ Medical College
and Research Hospital, Bengaluru, Karnataka, India

Corresponding Author: Manisha V Ramani, Department of Obstetrics and Gynaecology, MVJ Medical College and Research Hospital, Bengaluru, Karnataka, India, Phone: +91 9958849975, e-mail: manisha. ramani21@gmail.com

How to cite this article: Ramani MV, Saxena RK, Indrani C. Bowel Perforation due to COVID-19: A Mini Review. J South Asian Feder Obst Gynae 2020;12(6):427-431.

Source of support: Nil

Conflict of interest: None

An unusual presentation of bowel perforation in a patient who had recently undergone cesarean delivery $(C D)$ generated heated discussion in the department and this prompted us to report this mini review of bowel perforations attributed to coronavirus.

\section{CASE}

Our patient, a 19-year-old primigravida, underwent emergency CD under spinal anesthesia due to arrest of dilatation. A Pfannenstiel incision was made and the peritoneum was opened by blunt finger dissection. A live healthy baby girl was delivered by Kerr's incision. The uterus was exteriorized and closed in two layers. Hemostasis was ensured and the abdomen was closed in layers; rectus sheath was closed with polyglactin 910 suture. The skin was closed with subcuticular poliglecaprone 25 sutures. This being a primary $C D$, there were no adhesions, and no other intraoperative difficulty was encountered. Postoperatively patient made an uneventful recovery. Bowel sounds were confirmed, and the patient was started on oral 
fluids 6 hours after surgery. Normal diet was started the next day and she passed motion on the third postoperative day. The patient remained asymptomatic and was discharged on fifth postoperative day as per departmental protocol.

On the 10th postoperative day, the patient presented to the emergency department at 10 p.m., with complaints of vomiting of 2 days duration, loose stools for 1 day, and acute epigastric pain of 1-hour duration. This was followed by copious thick greenish discharge from the cesarean incision site, and this bilious discharge was nonfoul-smelling (Fig. 1). The patient was hemodynamically unstable. Her pulse rate was 140 beats per minute with cold clammy peripheries and blood pressure of $80 / 50 \mathrm{~mm} \mathrm{Hg}$. The patient was dyspneic with respiratory rate of 70 per minute with saturation of $85 \%$ on room air. No urine was drained on bladder catheterization. The patient was immediately shifted to the intensive care unit (ICU) and started on noradrenaline infusion at $5 \mathrm{~mL} /$ hour. Initial blood investigations revealed impaired renal function test with creatinine of $2.9 \mathrm{mg} / \mathrm{dL}$ and total leucocyte count of $23000 / \mathrm{mm}^{3}$. Serum electrolytes revealed hypokalemia with potassium 1.9 $\mathrm{mEq} / \mathrm{L}$, and arterial blood gases showed severe metabolic acidosis with respiratory alkalosis. A provisional diagnosis of septic shock secondary to bowel injury was made.

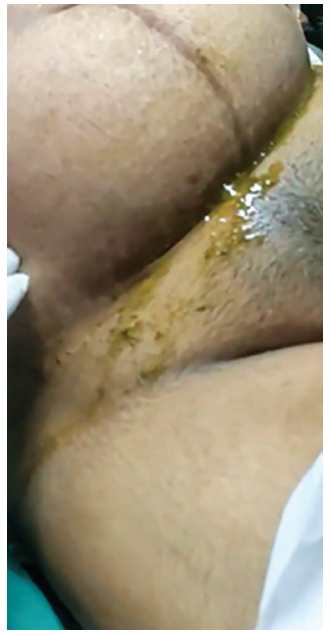

Fig. 1: Wound site with bilious discharge
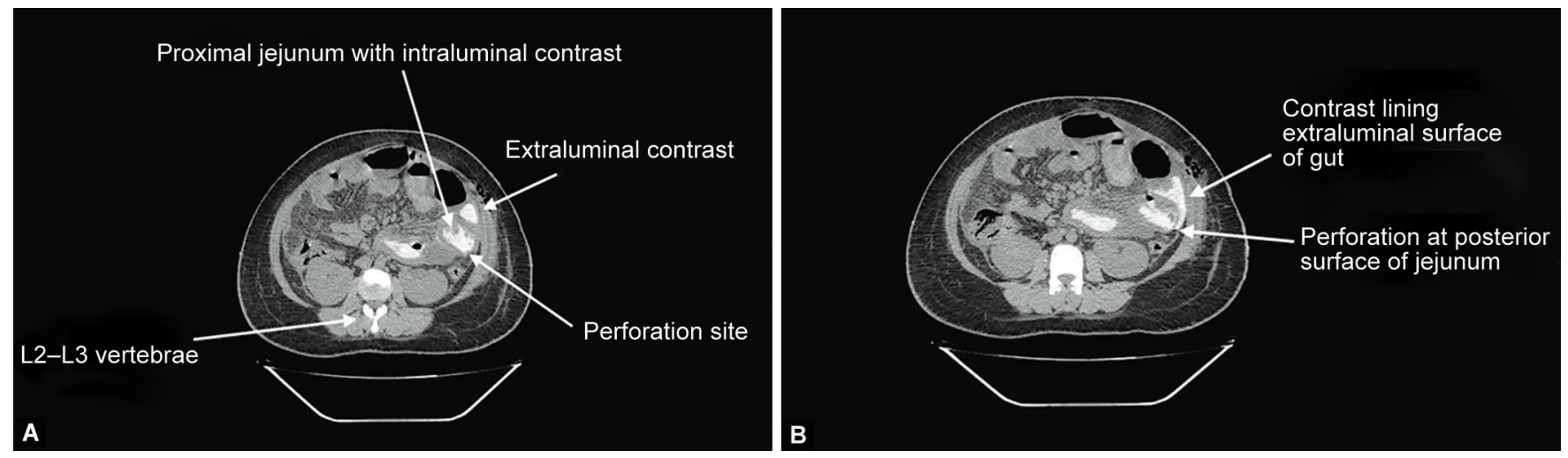

Fig. 2A and B: CT scan images showing perforation of proximal jejunum

With initial resuscitation, the patient's condition stabilized and an oral contrast computed tomography (CT) was done, which revealed a proximal jejunal perforation (Fig. 2). The patient was taken up for laparotomy under general anesthesia, which revealed a $1.5-\mathrm{cm}$ linear perforation in the proximal jejunum, $20 \mathrm{~cm}$ from duodenojejunal junction (Fig. 3). Bowel repair was done by the surgical team and two intraperitoneal drains (subhepatic and pelvic) were placed. In view of her poor general condition, the patient continued to be intubated and on ventilator support in ICU. On the first postoperative day of laparotomy, the intraperitoneal drain was minimal, but the patient developed high-grade fever of $105^{\circ} \mathrm{F}$. This prompted the investigation of a nasopharyngeal swab for RT-PCR test for COVID-19, which was reported to be positive. Twenty-four hours after the laparotomy, the intraperitoneal drains started draining copious bilious discharge $400-500 \mathrm{~mL}$ per/day. Thereafter, the general condition of the patient continued to deteriorate, and she expired on the third postoperative day.

\section{Discussion and Review}

The initial clinical presentation of the above case was confusing, and the discussion centered primarily around the etiology of the

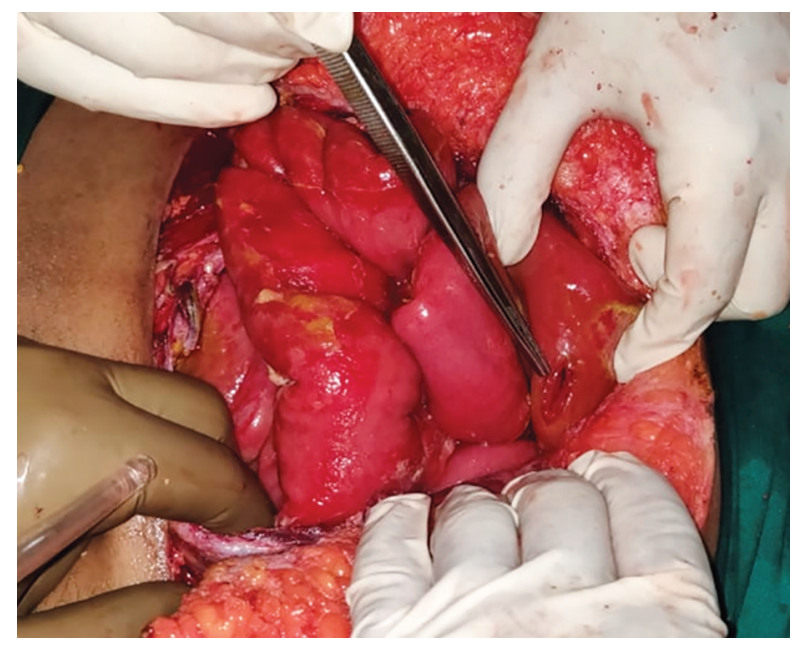

Fig. 3: Jejunal perforation 
intestinal perforation. In view of the recent history of surgical procedure, the obvious conclusion was an iatrogenic bowel injury during $C D$. However, the uneventful postoperative recovery of the patient after CD negates this theory. Confirmation of COVID-19 infection of the patient brought forth the possibility of extrapulmonary affection and the jejunal perforation. However, intestinal perforation in COVID-19 infection is an uncommon finding reported only in few case reports. Another differential diagnosis considered was the possibility of a free spontaneous jejunal perforation. The rarity of such perforations and the absence of any precipitating etiology prompted us to keep it as the last differential diagnosis.

\section{latrogenic Bowel Injury}

Since the patient had undergone $C D$ just 10 days prior, the initial diagnosis of intraoperative bowel injury was a strong contender for the patient's clinical presentation. However, the location of the perforation in the proximal jejunum created doubt about the etiology of the bowel injury. First, the cesarean was done through a Pfannenstiel incision, which along with the presence of gravid uterus makes access to proximal jejunum very difficult. Although bowel injuries are rare during $C D$, the commonly injured segment of the bowel is the colon, given the anatomic location near the surgical site. ${ }^{7}$ Second, this was a primary cesarean and the first abdominal surgery for the patient; hence, no bowel adhesions were encountered during surgery. Finally, the patient had an uneventful postoperative recovery after CD. Mesdaghinia et al. reported that mean time of diagnosis of an intraoperative bowel injury was $2.8 \pm 0.9$ days. ${ }^{7}$ Our patient was in the hospital till the fifth postoperative day, and she was taking a normal diet and had a normal bowel movement before discharge from the hospital. This would not have been possible if there was an intraoperative bowel injury or perforation. Delayed presentation due to thermal injury as a result of conductive cautery burn was also considered. Pathophysiology of such perforation invariably involves gross tissue necrosis. ${ }^{8}$ The surgical team did not observe any tissue necrosis around the perforation site.

\section{COVID-19}

Given the fact that COVID-19 infection is relatively new with ongoing research into the diverse presentation and progression of the disease, it made us to consider coronavirus as a plausible explanation for the patient's condition. The history of loose stools and vomiting followed by sudden severe epigastric pain points to the probable timing of jejunal perforation in our patient.

The currently available literature documents bowel perforation as a significant complication in COVID-19 patients. Neto et al. reported a case of an 80-year-old female patient with a history of dry cough and fever for 10 days. She presented with tachypnea, low oxygen saturation, and diffuse abdominal pain and stiffness. CT revealed ground-glass appearance of bilateral lungs and extensive pneumoperitoneum. The patient was taken up for exploratory laparotomy after resuscitative measures, which revealed reduced visceral blood flow to the bowel with four punctate lesions on the sigmoid colon with fecal matter draining in the peritoneal cavity. A rectosigmoidectomy was done followed by terminal colostomy, but the patient succumbed to her illness on postoperative day 2 due to refractory septic shock. ${ }^{9}$

Another case of bowel perforation was documented in a 53-year-old man recovering from COVID-19 pneumonia. The patient was treated for ARDS and was in ICU for 6 days. Three days after shifting out from ICU, the patient complained of diarrhea and abdominal pain with marked abdominal distention. Plain $X$-ray and abdominal CT scan revealed air in the intraperitoneal cavity, distended large bowel, and perforation of ascending colon. Emergency laparotomy followed by colonic resection was performed. Histological examination revealed transmural granulocytic inflammation with mucosal and submucosal edema. No thrombosis or vasculitis was observed. The authors suggested that the colonic perforation was due to overdistension of the bowel. Recovery was also complicated by new-onset fever managed with parenteral antibiotics. The patient had a full recovery and was discharged on postoperative day $18 .^{10}$

A similar case was observed in a 47-year-old male patient who after two weeks of ICU stay for acute respiratory illness due to COVID-19 presented with abdominal pain and distention. CT scan revealed small bowel ischemia with perforation. However, his mesenteric vessels were noted to be patent. On exploration of abdomen, bowel was found to be necrotic from the ligament of Treitz, up till the transverse colon with perforation of the distal ileum. The distribution of necrotic areas was varied with some areas having circumferential involvement whereas some others were having patchy involvement along the antimesenteric border of the small bowel. The patent mesenteric vessels and presence of necrosis on the antimesenteric border suggest microvascular thrombosis as hypercoagulability is an established feature of COVID-19. ${ }^{2}$ Dick et al. also reported findings of proximal gastrointestinal perforation in a case of severe COVID-19 pneumonia. ${ }^{11}$

Bhayana et al. observed bowel abnormalities in $31 \%$ of patients admitted in ICU with COVID-19. The bowel abnormalities included bowel thickening, pneumatosis or portal venous gas, bowel ischemia, and perforation. ${ }^{12}$

Recent studies into the pathophysiology of coronavirus suggest that coagulopathy, both venous and arterial thromboembolisms, is the most severe sequela of the disease and it is associated with poor prognostic outcome. ${ }^{13-16}$ The theory of inflammatory thrombosis is consistent with the laboratory data of critically ill COVID-19 patients, who have elevated levels of C-reactive proteins, IL-6, fibrinogen, D-dimers, ferritin, and lactate dehydrogenase. ${ }^{17}$ Thrombosis in the small vessels of the bowel leads to ischemia and perforation. ${ }^{12}$ Patients who are inherently at higher risk of endothelial damage as in obesity, hypertension, and diabetes tend to progress to severe forms of COVID-19 infection and have a poorer prognosis. ${ }^{18}$

The review of the literature suggests that COVID-19 could be a distinct possibility for the jejunal perforation in our patient. Our patient was in the postpartum period, a hypercoagulable state. The history of vomiting and loose stools and acute epigastric pain are all suggestive of gastrointestinal affection of COVID-19. On the second postoperative day after jejunal perforation repair, the abdominal drains again had copious bilious discharge, suggesting either breakdown of existing repair or a new spontaneous perforation due to severe COVID-19 infection.

It may be argued that our patient did not have any respiratory symptoms suggestive of COVID-19 infection at the time of admission. This is not surprising as Lu Lin et al. and Jin et al. have reported that up to $28.38 \%$ of COVID-19 patients who present with gastrointestinal symptoms at the onset of illness lack the classical respiratory symptoms. ${ }^{1,19}$ A metanalysis conducted by Mao et al. observed that almost $10 \%$ of patients present with only gastrointestinal symptoms without any respiratory features. Patients with only gastrointestinal symptoms due to COVID-19 may have an insidious progression of the disease. Early diagnosis is a clinical challenge..$^{5,20}$ 


\section{Spontaneous Free Small Bowel Perforation}

Spontaneous free perforation of the small bowel is a fairly uncommon diagnosis. Causes for the same are listed in Table 1.

A thorough history from the next of kin ruled out infectious causes like tuberculosis (TB) and typhoid fever. Intraoperative surgical findings did not support the possibility of TB as a cause of perforation. Abdominal TB mainly affects the ileocecal junction. These perforations are not directly due to tuberculous ulcers but rather "blow-out." Perforations occur as a result of distension secondary to distal obstructions. ${ }^{21}$ No such obstruction was evidenced during the laparotomy of our patient. Perforations as a sequela of enteric fever most commonly affect the terminal ileum. Jain et al. reported that almost $97 \%$ of enteric perforations occur in the ileum. ${ }^{22}$ Our patient had no history suggestive of enteric fever.

The only remote possibility that could be considered may be the initial presentation of Crohn's disease or a drug-mediated intestinal injury. In Crohn's disease, the most common site for perforation is the ileum but rarely, jejunum may also be involved. ${ }^{23}$ Interestingly, free bowel perforation may be the first presentation of Crohn's disease. However, the intraoperative status of the bowel negates this possibility as no gross features of Crohn's disease were identified.

Nonsteroidal anti-inflammatory drug (NSAID)-induced nonspecific ulcers may also cause free spontaneous perforations. ${ }^{24,25}$

Table 1: Causes for small bowel perforation

\begin{tabular}{|c|c|}
\hline Causes of bowel perforation & Specific forms \\
\hline \multicolumn{2}{|l|}{ Traumatic } \\
\hline Trauma & Shot, stab, blunt injury \\
\hline Foreign body & Toothpick, fishbone \\
\hline latrogenic & Laparoscopy, laparotomy \\
\hline \multicolumn{2}{|l|}{ Free perforations } \\
\hline \multicolumn{2}{|l|}{ Irradiation } \\
\hline \multirow[t]{5}{*}{ Drugs } & NSAIDs \\
\hline & Steroids \\
\hline & Oral contraceptive pills \\
\hline & Cytotoxic chemotherapy \\
\hline & Cocaine \\
\hline \multirow[t]{3}{*}{ Vascular problems } & Wegener's granulomatosis \\
\hline & Giant cell arteritis \\
\hline & Buerger's disease \\
\hline Congenital anomalies & Meckel's diverticulum \\
\hline \multirow[t]{6}{*}{ Inflammatory disease } & Inflammatory disease \\
\hline & Crohn's disease \\
\hline & Tuberculosis \\
\hline & Typhoid fever \\
\hline & Ascariasis \\
\hline & Cytomegalovirus \\
\hline \multirow[t]{4}{*}{ Tumors } & Lymphoma \\
\hline & Adenocarcinoma \\
\hline & Angiosarcoma \\
\hline & $\begin{array}{l}\text { Metastatic melanoma, mesothelioma, } \\
\text { lung cancer, breast cancer }\end{array}$ \\
\hline Graft vs host reaction & \\
\hline
\end{tabular}

In India, NSAIDs and steroids are over-the-counter drugs and can be availed without prescription. The literature suggests that such perforations are more common in the duodenum..$^{25}$ The next of kin denied routine use of painkillers by the patient after $C D$ or any other drugs by the patient.

In light of the above-mentioned literature review, the general consensus was that our patient had developed bowel perforation due to COVID-19 infection.

\section{Conclusion}

The dramatic presentation of bowel perforation in our patient after CD, caught us off-guard, and a positive COVID-19 report came as a complete surprise to the treating team. The unusual clinical presentation of patients with extrapulmonary symptoms of COVID-19 infection is a diagnostic dilemma. Its unpredictable course demands a high index of suspicion and a low threshold for diagnostic testing, so that early treatment can be initiated to prevent the devastating consequences in these patients.

\section{References}

1. Lin L, Jiang X, Zhang Z, et al. Gastrointestinal symptoms of 95 cases with SARS-CoV-2 infection. Gut 2020;69(6):997-1001. DOI: 10.1136/ gutjnl-2020-321013.

2. Gartland RM, Velmahos GC. Bowel necrosis in the setting of COVID19 [published online ahead of print, 2020 May 3]. J Gastrointest Surg 2020;1-2. DOI: 10.1007/s11605-020-04632-4.

3. Wu F, Zhao S, Yu B, et al. A new coronavirus associated with human respiratory disease in China. Nature 2020;579:265-269. DOI: 10.1038/ s41586-020-2008-3.

4. Zhang $H$, Kang Z, Gong $H$, et al. The digestive system is a potential route of 2019-nCov infection: a bioinformatics analysis based on single-cell transcriptomes. BioRxiv 927806. Post online Jan 31. DOI: 10.1101/2020.01.30.927806.

5. Parry $A H$, Wani $A H$, Yaseen $M$. Acute mesenteric ischemia in severe coronavirus-19 (COVID-19): possible mechanisms and diagnostic pathway. Letter to editor. Acad Radiol 2020;27(8):1190. DOI: 10.1016/j. acra.2020.05.016.

6. Singhania N, Bansal S, Nimmatoori DP, et al. Current overview on hypercoagulability in COVID-19. Am J Cardiovasc Drugs 2020;20:393403. DOI: 10.1007/s40256-020-00431-z.

7. Mesdaghinia E, Abedzadeh-Kalahroudi M, Hedayati M, et al. latrogenic gastrointestinal injuries during obstetrical and gynecological operation. Arch Trauma Res 2013;2(2):81-84. DOI: 10.5812/atr.12088.

8. Stany MP, Farley JH. Complications of gynecologic surgery. Surg Clin North Am 2008;88:343-359. DOI: 10.1016/j.suc.2007.12.004.

9. Corrêa Neto I, Viana K, Silva M, et al. Perforated acute abdomen in a patient with COVID-19: an atypical manifestation of the disease. J Coloproctol 2020;40(3):269-272. DOI: 10.1016/j.jcol.2020.05.011.

10. De Nardi P, Parolini D, Ripa M, et al. Bowel perforation in a COVID-19 patient: case report. Int J Colorectal Dis 2020;35(9):1797-1800. DOI: 10.1007/s00384-020-03627-6.

11. Kangas-Dick A, Prien C, Rojas K, et al. Gastrointestinal perforation in a critically ill patient with COVID-19 pneumonia. SAGE Open Med Case Rep 2020;8:1-4. DOI: 10.1177/2050313X20940570.

12. Bhayana R, Som A, Li M, et al. Abdominal imaging findings in COVID19: preliminary observations. Radiology 2020;297(1):207-215. DOI: 10.1148/radiol.2020201908.

13. Zhou F, Yu T, Du R, et al. Clinical course and risk factors for mortality of adult in patients with COVID-19 in Wuhan, China: a retrospective cohort study. Lancet 2020;395(10229):1054-1062. DOI: 10.1016/S01406736(20)30566-3.

14. Guan WJ, Ni ZY, Hu Y, et al. Clinical characteristics of coronavirus disease 2019 in China. N Engl J Med 2020;382(18):1708-1720. DOI: 10.1056/NEJMoa2002032. 
15. Huang C, Wang Y, Li X, et al. Clinical features of patients infected with 2019 novel coronavirus in Wuhan, China. Lancet 2020;395(10223):497506. DOI: 10.1016/S0140-6736(20)30183-5.

16. Tang N, Li D, Wang $X$, et al. Abnormal coagulation parameters are associated with poor prognosis in patients with novel coronavirus pneumonia.JThromb Haemost 2020;18(4):844-847.DOI: 10.1111/jth.14768.

17. Panigada $M$, Bottino N, Tagliabue P, et al. Hypercoagulability of COVID19 patients in intensive care unit. A report of thromboelastography findings and other parameters of hemostasis. J Thromb Haemost 2020;18(7):1738-1742. DOI: 10.1111/jth.14850.

18. Varga Z, Flammer AJ, Steiger $P$, et al. Endothelial cell infection and endotheliitis in COVID-19. Lancet 2020;395(10234):1417-1418. DOI: 10.1016/S0140-6736(20)30937-5.

19. Jin X, Lian J, Hu J, et al. Epidemiological, clinical and virological characteristics of 74 cases of coronavirus-infected disease 2019 (COVID-19) with gastrointestinal symptoms. Gut 2020;69(6):10021009. DOI: 10.1136/gutjnl-2020-320926.

20. Mao R, Qiu Y, He JS, et al. Manifestations and prognosis of gastrointestinal and liver involvement in patients with
COVID-19: a systematic review and meta-analysis. Lancet Gastroenterol Hepatol 2020;5(7):667-678. DOI: 10.1016/S24681253(20)30126-6.

21. Gupta S, Jayant M, Kaushik R. Free tubercular perforation of the ileum. World J Emerg Med 2013;4(3):235-236. DOI: 10.5847/ wjem.j.issn.1920-8642.2013.03.015.

22. Jain S, Meena LN, Ram P. Surgical management and prognosis of perforation secondary to typhoid fever. Trop Gastroenterol 2016;37(2):123-128. DOI: 10.7869/tg.336.

23. Freeman HJ. Spontaneous free perforation of the small intestine in Crohn's disease. Can J Gastroenterol 2002;16:23-27. DOI: $10.1155 / 2002 / 284958$.

24. Sakaguchi T, Tokuhara K, Nakatani K, et al. Laparoscopic management for spontaneous jejunal perforation caused by nonspecific ulcer: a case report. Int J Surg Case Rep 2017;39:309-312. DOI: 10.1016/j. ijscr.2017.08.048.

25. Nahar S, Ranjan A. Observational study of small bowel perforation in a tertiary care hospital. Int Surg J 2017;4(8):2746-2750. DOI: 10.18203/2349-2902.isj20173411. 